\title{
Abstract: Does Bone Suppression and Lung Detection Improve Chest Disease Classification?
}

\author{
Ivo M. Baltruschat ${ }^{1,2,3,4}$, Leonhard A. Steinmeister ${ }^{1,3}$, Harald Ittrich $^{1}$, \\ Gerhard Adam ${ }^{1}$, Hannes Nickisch ${ }^{4}$, Axel Saalbach ${ }^{4}$, Jens von Berg ${ }^{4}$, \\ Michael Grass ${ }^{4}$, Tobias Knopp ${ }^{1,2}$ \\ ${ }^{1}$ Department for Diagnostic and Interventional Radiology and Nuclear Medicine, Uni- \\ versity Medical Center Hamburg-Eppendorf, Germany \\ ${ }^{2}$ Institute for Biomedical Imaging, Hamburg University of Technology, Germany \\ ${ }^{3}$ DAISYLabs, Forschungszentrum Medizintechnik Hamburg, Germany \\ ${ }^{4}$ Philips Research, Hamburg, Germany \\ i.baltruschat@uke.de
}

Chest radiography is the most common clinical examination type. To improve the quality of patient care and to reduce workload, researchers started developing methods for automatic pathology classification. In our paper [1], we investigate the effect of advanced image processing techniques - initially developed to support radiologists - on the performance of deep learning techniques.

First, we employ bone suppression, an algorithm to artificially remove the rib cage in chest X-ray images. Secondly, we use automatic lung field detection to crop images to the lung area. Furthermore, we consider the combination of both. For convolutional neural network (CNN) training and evaluation, DICOM images from the Indiana dataset (Open-I [2]), were examined by two expert radiologists and annotated with respect to eight different pathologies. We pretrain our CNN on the largest publicly available X-Ray dataset (ChestX-ray14) and fine-tune it by using the DICOM data.

In a five-times re-sampling scheme, we use receiver operating characteristic (ROC) statistics to evaluate the effect of the pre-processing approaches. Using a convolutional neural network $(\mathrm{CNN})$, optimized for X-ray analysis, we achieve a good performance with respect to all pathologies on average. While, the combination of bone suppression and lung field detection improves slightly the average ROC area from $0.891 \pm 0.013$ to $0.906 \pm 0.012$. Contrary, for selected pathologies, a substantial improvement can be reported i.e. for mass the area under the ROC curve increased by $9.95 \%$. The ensemble with pre-processed trained models yields the best overall results with $0.912 \pm 0.011$ AUC on average.

\section{References}

1. Baltruschat IM, Steinmeister LA, Ittrich H, et al. When does bone suppression and lung field segmentation improve chest X-ray disease classification? Proc ISBI. 2019;.

2. Demner-Fushman D, Kohli MD, et al. Preparing a collection of radiology examinations for distribution and retrieval. J Am Med Inform Assoc. 2015; p. 304-310. 